\title{
Dark energy rips cosmos and agencies
}

\section{An international space mission to study an astronomical mystery is foundering.}

A once-favoured space probe to study dark energy is struggling to get off the ground, as three agencies in the United States and Europe tussle over the details of a potential international mission.

The rise and fall this year of the Joint Dark Energy Mission (JDEM) - a satellite meant to pin down the repulsive force that is accelerating the Universe's expansion - is partly due to strife between two US agencies, NASA and the Department of Energy (DOE), and a third potential partner, the European Space Agency (ESA). In addition, scientists working on the JDEM designs have not presented a unified front, owing to disagreements over the best observational method to use (see 'Hunting for dark energy') - at a time when an influential astrophysics panel is about to prioritize the next decade's best and most organized missions.

"This is an example of a satellite blowing up before it gets built," says Bob Nichol, an astrophysicist at the University of Portsmouth, $\mathrm{UK}$, who is working on the European design concept.

Dark energy is a fudge factor for a force that has the upper hand on gravity, pushing the Universe to accelerate at an ever-faster rate. The effect was announced in 1998 after astronomers precisely measured the distances to supernovae in other galaxies. But the cause remains baffling. "It is perhaps the biggest mystery of our time," says Neil Gehrels, JDEM project scientist at the Goddard Space Flight Center in Greenbelt, Maryland. "It determines the fate of the Universe." Depending on how strong it turns out to be, dark energy could dissipate the Universe into darkness, shred it in a 'big rip' or even reverse itself, allying with gravity to create a 'big crunch'.
JDEM was meant to figure this out, and the mission had early momentum. It got a political boost from a 2007 study by the National Research Council that ranked a dark-energy probe as the top priority for studying deep cosmological questions. Then, to forge a scientific consensus, in September 2008 NASA and the DOE brought together three design teams that had been competing to fashion a mission that could potentially accommodate all of them (see Nature 455, 577; 2008).

\section{Hunting for dark energy}

Scientists are arguing over three space-based approaches to measure dark energy.

Supernovae Measuring distances to supernovae was the first, and best-understood, way to probe dark energy, but also has the most limitations. It assumes that all supernovae in the early Universe exploded with the same characteristic brightness.
Baryon acoustic oscillations Sound waves from the Big Bang imprinted ripples in the distribution of galaxies. By comparing the size of the ripples in early galaxy clusters with those in clusters that formed later in the Universe, astronomers can deduce the effect of dark energy. But finding enough young clusters big enough to work with is a challenge.
Weak lensing This looks for tiny distortions in the shapes of galaxies caused by intervening dark matter, which varies with cosmic time and relates to dark energy. It requires images of many galaxies from surveys, which means that such a mission would need to operate differently from one optimized for baryon acoustic oscillations. 
Two months later, the agencies inked an agreement in which NASA would lead the mission. The DOE has said it would contribute roughly one-quarter of the cost.

But price was a problem. Separately, each of the three teams had missions with price tags of more than US\$1 billion; combined, the JDEM was just as expensive, if not more so. NASA's astrophysics division had always wanted something smaller and cheaper. But "how much dark energy can you buy for $\$ 600$ million?" asked Jon Morse, director of NASA's astrophysics division, at an advisory meeting this month. "No one seems to want to answer that question."

So NASA went to ESA, which had been pursuing its approach, called Euclid, for years. By January, it looked as if ESA - and its budgetary resources - were on board (see Nature doi:10.1038/news.2009.38; 2009).

\section{Downgraded}

But when NASA told DOE officials that the Europeans would be building scientific instruments that the DOE had planned to make itself, the energy agency baulked. The DOE-NASA agreement specifies that the DOE would build a "major scientific instrument". Yet in negotiations through the winter, the department was offered minor-league work that some there considered insulting.

"I don't disagree that there were unhappy scientists on all three sides," says Gehrels. But no division of duties was set in stone then, he says. And he points out that very few multi-agency missions are smooth; for instance, the \$700-million Fermi Gamma-ray Space Telescope, with contributions from the DOE and NASA, went through a difficult birthing process for more than a decade before being launched last year. At a physics advisory meeting last week in Washington DC, William Brinkman, the DOE's director for the Office of Science, said of JDEM: "It's not an uncontroversial situation."

DOE officials and scientists were so unhappy that, for a few months this spring, they broke off from the mission. Some, at the Lawrence Berkeley National Laboratory in California, instead proposed a ground-based dark-energy survey called BigBOSS. In presentations in June, the team claimed that BigBOSS would rival JDEM's results for an overall cost of just $\$ 85$ million.

Meanwhile, NASA and ESA ploughed ahead with a design that became known as the International Dark Energy Cosmology Survey (IDECS). In April, this partnership also ended because the merger wasn't happening quickly enough. In Europe, the Euclid team was told to resume work on its 1.2-metre space telescope, Nichol says, in preparation for a triage in February 2010 under ESA's 'Cosmic Visions' competition.

On the US side, three design concepts remain in play. The \$1.6-billion IDECS design has two types of detectors and would use all three observational methods on a 1.5-metre telescope. A \$1.2-billion 'Omega' design would use only one detector and sacrifice some capabilities. Gehrels says that his team has now been tasked to look at a third concept, with a price cap of $\$ 850$ million, that will require a smaller telescope and cut the probe's capabilities.

Meanwhile, as the space-based mission ideas stall, ground-based astronomers are making strides in pinning down uncertainties in a key dark-energy parameter that determines whether the force is a constant or changes with time. One of many recent studies, which added more than 100 newly observed supernovae to existing studies, suggests that it is a constant with a precision of $7 \%$ (M. Hicken et al. Astrophys. J. 700, 1097-1140; 2009).

Simon White, director of the Max Planck Institute for Astrophysics in Garching, Germany, questions whether it is worthwhile to spend a billion dollars just to show, with more precision, that dark energy is a constant. "There's nothing to look for," says White. "It's fairly close to a cosmological constant, and the question [for JDEM] is: is it very, very close to a cosmological constant?"

But all the recent studies that seem to be honing in on a cosmological constant make the assumption that it is constant in time, says Rocky Kolb, a theorist at the University of Chicago in Illinois.

To achieve precision and to test how dark energy has varied in time, Kolb says, going to space is necessary. Both possibilities - a constant, or a changing dark energy — are equally ugly to theorists, who have few explanations for either scenario.

Kolb notes that there is a third possible result. Several ground-based microwave telescopes, such as the South Pole Telescope, are tracking how the structure of very distant galaxy clusters grew in the early Universe under the influence of gravity. If these results, expected soon, do not agree with the measurements of the expansion history of space - the measurements made by methods used by JDEM - it would indicate that something is wrong with general relativity.

But JDEM may not get to make these comparisons. NASA and the DOE will not commit to any of the designs until after the community survey weighs in in spring 2010. "We'll wait for the decadal survey," Morse said earlier this month, "to see if we have a mission."

Eric Hand 